hautnah $2021 \cdot 20: 98-107$

https://doi.org/10.1007/s12326-021-00439-5

Angenommen: 26. März 2021

Online publiziert:9. April 2021

(c) Der/die Autor(en) 2021

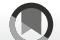

Check tor
updates

\section{P. M. Heil}

Kollagenosen-Ambulanz, Universitätsklinik für Dermatologie, Medizinische Universität Wien, Wien, Österreich

\title{
Mehr als nur der Schmetterling - ein Leitfaden durch die Vielfalt des kutanen Lupus erythematodes
}

\author{
Pathogenese, Klinik, Therapie
}

Der Lupus erythematodes (LE) ist eine entzündliche Autoimmunerkrankung, bei der einerseits ausschließlich die Haut in unterschiedlicher Art und Weise betroffen sein kann (kutaner LE, $C L E$ ), andererseits aber auch andere immunserologische (z.B. Autoantikörper) oder klinische Befunde abseits der Haut (z.B. Serositis, Nephritis etc.) positiv sein können: Diese Konstellation nennt man einen systemischen Lupus erythematodes (SLE, Klassifikationskriterien s. unten). Die Inzidenz des CLE liegt bei ca. 4/100.000 [1]. $75 \%$ der SLE-Patienten entwickeln im Laufe ihrer Erkrankung eine der CLE-Formen [2].

\section{Pathogenese}

Die exakte pathogenetische Kette des LE ist nicht im Detail geklärt, wohl aber sind wichtige Puzzlesteine bekannt, die wesentlich sind, um das autoaggressive Verhalten des Immunsystems in Gang zu setzen.

Das entzündliche Infiltrat des CLE ist dominiert von T-Zellen (CD3+ Infiltrat), insbesondere von zytotoxischen T-Zellen $[3,4]$, und attackiert die dermoepidermale Junktionszone. Einerseits durch diese entzündliche Attacke, andererseits auch durch exogene Einflüsse (starke UV-Exposition) kommt es zur Schädigung von Keratinozyten und Freilegung sonst intrazellulär gelegener Antigene, die nun erkannt und attackieret werden können [5]. Nach Sonnenexposition und Aktivie- rung der autoimmunen Reaktion kommt es typischerweise erst 1-2 Wochen später zu einer Verschlechterung bestehender oder Auftreten neuer CLE-Läsionen, da die oben geschilderte orchestrierte Immunreaktion Zeit benötigt, um voll in Gang zu kommen.

Hinzu kommt die Funktion von B-Zellen. Zum einen sind diese Antigen-Präsentatoren („antigen-presenting cells", APCs) für T-Zellen, zum anderen sind sie Quelle der Plasmazellen, welche Autoantikörper produzieren, deren Zielantigene nach Zellschaden (s. oben) offenliegen. Dazu kommt, dass Keratinozyten nicht nur Ziel, sondern auch Akteur der Entzündungsreaktion sein können, indem sie Typ-I- und Typ-IIIInterferone (IFN $\kappa$ und IFN $\lambda$ ) sowie Interferon-abhängige Botenstoffe (z.B. CXCL10) produzieren [6-9]. Letzteres attrahiert CXCR3-positive T-Zellen, was zur Nekroptose von Keratinozyten führt $[10,11]$.

\section{) Keratinozyten sind nicht nur Ziel, sondern können auch Akteur der Entzündungsreaktion sein}

Wenngleich der LE als eine Erkrankung angesehen wird, dessen Pathogenese prototypisch für das erworbene Immunsystem (zytotoxisches T-ZellInfiltrat, Autoantikörper) ist, ist das angeborene Immunsystem jedoch ebenso involviert. So können zirkulierende Immunkomplexe (z.B. bestehend aus Autoantikörpern und RNA oder DNA) von plasmazytoid-dendritischen Zellen (CD123+HLADR+BDCA-2+) aufgenommen werden und mittels Aktivierung der endosomalen Rezeptoren TLR7 und TLR9 die Produktion von Typ-IInterferon auslösen [12]. Der LE wird als prototypisch für eine Erkrankung mit Interferon-Signatur angesehen.

Ein weiteres interessantes Phänomen des angeborenen Immunsystems in der Pathogenese des SLE sind die "neutrophil extracellular traps“ (NETs), welche nicht nur beim SLE, sondern auch beim CLE beschrieben wurden [13]. NETs dienen eigentlich der Abwehr von Pathogenen (z. B. Bakterien), können jedoch auch DNA binden und dadurch Autoimmunität propagieren.

Die höhere Prävalenz von Frauen gegenüber Männern sowohl beim SLE [14] als auch beim CLE [15] ist lange bekannt, Östrogene spielen hier die wesentliche Rolle [16]. In den letzten Jahren konnten weitere, für einen LE prädisponierende Faktoren eruiert werden.

Es wurde eine Vielzahl von Polymorphismen und genetischen Assoziationen beschrieben [17], bis dato sind jedoch nur wenige monogenetische Varianten des CLE bekannt (TREX-1-Mutationen in TREX-1, SAMHD1 sowie STING; [18-20]). 


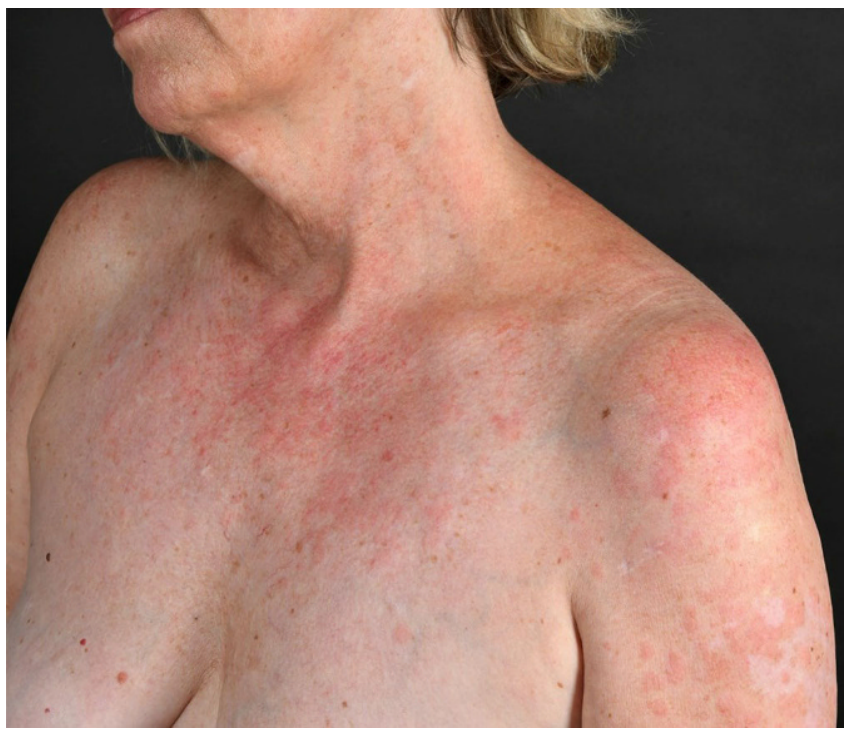

Abb. $1<$ CLE-

Schub ausgelöst durch psychosoziale Belastung (terminale Erkrankung einer engen Bezugsperson)

Rauchen ist mittlerweile als Risikofaktor für schwerere Verläufe des CLE etabliert [21].

Ebenso können Medikamente einen Lupus erythematodes provozieren („drug-induced lupus“, DIL; [22-24]). $\mathrm{Zu}$ den Auslösern gehören Procainamid, Hydralazin, Minozyklin, Terbinafin, Sulfasalazin, Carbamazepin, die Gruppe der Statine, der KalziumkanalBlocker und ACE-Hemmer und Checkpoint-Inhibitoren. Ebenso wurde in Fallberichten dokumentiert, dass ältere, chimär human-murine TNF- $\alpha$-Blocker (Infliximab) bzw. modernere, humanisierte (Certolizumab) bzw. voll-humanrekombinante (Adalimumab) TNF- $\alpha$ Blocker einen DIL hervorrufen können.

\section{》) Rauchen ist als Risikofaktor für schwerere Verläufe des CLE etabliert}

Zur Aktivität eines LE können zudem bakterielle [25] und virale [26] Infektionen beitragen; speziell auf letzteren liegt im Rahmen der COVID-19-Pandemie erneut ein spezielles Augenmerk [27].

Nicht zuletzt sei darauf hingewiesen, dass psychosoziale Stressoren (z. B. Erkrankung oder Tod naher Bezugspersonen) zu LE-Schüben führen können ([28]; • Abb. 1).

\section{Klinik}

In der Abklärung eines Lupus erythematodes geht es aus dermatologischer Sicht immer um 2 Fragen:

1. Sind die Hautveränderungen, mit denen sich ein Patient vorstellt, ein CLE, und, falls ja, um welche CLESubform handelt es sich (s. unten)?

\section{Besteht zusätzlich ein SLE?}

In Bezug auf Hautveränderungen im Rahmen eines LE unterscheidet man spezifische Hautveränderungen, die nur beim LE vorkommen (CLE), von jenen Hautveränderungen, die unspezifisch sind und auch bei anderen Kollagenosen vorkommen können.

Die zwei wichtigsten Klassifikationen des CLE sind jene von Sontheimer [29] und Kuhn ([30]; - Tab. 1).

Man unterscheidet beim CLE einen akut-cutanen LE (ACLE), von einem subakut-cutanen LE (SCLE) sowie einem chronisch-cutanen LE (CCLE). Diese Einteilung orientiert sich einerseits an der Aktualität bzw. der Chronizität des Geschehens, andererseits an der Morphe der Veränderungen.

Ein isolierter Hautbefall (ohne Beteiligung innerer Organe bzw. ohne positive Immunserologie) tritt vorzugsweise als CCLE auf; die höchste Wahrscheinlichkeit, parallel zur Haut auch an einem SLE zu leiden, hat der ACLE. Es sollte einem jedoch bewusst sein, dass selbst im Fall eines SLE Hautherde auftreten kön-
Tab. 1 Klassifikation des kutanen Lupus erythematodes. (Mod. nach [29] und [30])

\begin{tabular}{|c|c|}
\hline \multirow[t]{4}{*}{$\begin{array}{l}\text { Akut-cutaner LE } \\
\text { (ACLE) }\end{array}$} & $\begin{array}{l}\text { Schmetterlingserythem } \\
\text { (,butterfly-rash“) }\end{array}$ \\
\hline & $\begin{array}{l}\text { Makulopapulöser } \\
\text { ACLE }\end{array}$ \\
\hline & TEN-like ACLE \\
\hline & Mucosal ACLE \\
\hline \multirow{2}{*}{$\begin{array}{l}\text { Subakut-cutaner LE } \\
\text { (SCLE) }\end{array}$} & Anulär \\
\hline & Papulosquamös \\
\hline \multirow[t]{6}{*}{$\begin{array}{l}\text { Chronisch-cutaner } \\
\text { LE (CCLE) }\end{array}$} & $\begin{array}{l}\text { Chronisch-diskoid } \\
\text { (CDLE), limitiert }\end{array}$ \\
\hline & $\begin{array}{l}\text { Chronisch-diskoid } \\
\text { (CDLE), disseminiert }\end{array}$ \\
\hline & $\begin{array}{l}\text { Lupus-Pannikulitis/ } \\
\text { Lupus profundus }\end{array}$ \\
\hline & Hypertropher CDLE \\
\hline & Chilblain-Lupus \\
\hline & Mucosal CCLE \\
\hline $\begin{array}{l}\text { Intermittierend- } \\
\text { cutaner LE (ICLE) }\end{array}$ & Lupus tumidus \\
\hline
\end{tabular}

nen, die von der Morphologie und vom Verlauf ein CCLE sind. Zudem können verschiedene CLE-Formen auch parallel auftreten (z.B. CCLE und ACLE).

Die häufigste Variante des kutanen LE ist der CCLE. Eine Subform ist der chronisch-diskoide Lupus erythematodes (CDLE), welcher in der Regel durch einen isolierten Befall der Haut ohne Zeichen einer Systembeteiligung (Immunserologie, Organbeteiligung) gekennzeichnet ist und bevorzugt an sonnenexponierten Arealen auftritt, durchaus typisch ist ein Befall der Ohrmuschel. Ist nur der Kopf betroffen, spricht man vom limitierten CDLE (• Abb. 2); ist auch der Rest des Körpers involviert, dann spricht man vom disseminierten CDLE (• Abb. 3). Die Erkrankung verläuft chronisch und in Schüben, wobei die Schübe durch UVExposition getriggert werden können. Die ohne Therapie über Monate und Jahre an Größe zunehmenden Herde beginnen als schuppende erythematöse Plaques, die mit der Zeit zentral einsinken und vernarben bzw. zu einer vernarbenden Alopezie führen können (• Abb.4). Durch die Vernarbung kann es mit der Zeit sogar zu Mutilationen kommen. Eine Sonderform des CDLE ist der hypertrophe CDLE (• Abb. 5), bei dem es durch eine ausgeprägte Hy- 


\section{P. M. Heil}

\section{Mehr als nur der Schmetterling - ein Leitfaden durch die Vielfalt des kutanen Lupus erythematodes. Pathogenese, Klinik, Therapie}

\section{Zusammenfassung}

Die vielen klinischen Varianten des kutanen Lupus erythematodes (CLE) können solitär oder im Rahmen eines systemischen Lupus erythematodes (SLE) auftreten, auf dessen Vorkommen regelmäßig gescreent werden muss. Neben dem weiblichen Geschlecht und genetischen Faktoren stellen Sonnenexposition, Rauchen und manche Medikamente Risikofaktoren dar. Die wichtigsten CLE-Formen sind der akutkutane LE (z. B. Schmetterlingserythem, generalisiert makulopapulös, enoral), der subakut-kutane LE (z. B. anuläre Form) und der chronisch-kutane LE (z. B. vernarbend diskoide Läsionen, Pannikulitis, Chilblain-LE). Die Diagnose beruht vor allem auf der Klinik und der Histopathologie, hinzu kommen autoimmunserologische Befunde und die direkte Immunfluoreszenz. Milde CLE-Formen können lokal therapiert werden. Reicht dies nicht aus, ist neben einem Steroidstoß Hydroxychloroquin die Systemtherapie der Wahl. Erweiterte therapeutische Optionen stellen Methotrexat, Retinoide, Dapson, Mycophenolat Mofetil, Azathioprin, Thalidomid, Belimumab und Rituximab dar. Alle CLETherapien sind off-label. Eine Aktualisierung der Impfungen sollte nach Möglichkeit vor Beginn einer Immunsuppression stattfinden. Zur Objektivierung des therapeutischen Ansprechens eines CLE empfiehlt sich das regelmäßige Scoring mittels RCLASI (Revised
CLE Disease Area and Severity Index). Präventiv ist Sonnenschutz (Cremen, Kleidung, Reiseziele) von höchster Wichtigkeit, da Sonnenexposition Schübe provozieren kann. Ein LE stellt keine Kontraindikation gegen eine Schwangerschaft (SS) dar, jedoch sollte diese nicht in einem Schub eintreten, da dies das Risiko für Fetus und Mutter erhöht. Therapeutisch kommen während einer SS v.a. Steroide, Hydroxychloroquin, Dapson und Azathioprin in Betracht.

\section{Schlüsselwörter}

Systemischer Lupus erythematodes · Autoimmunität · Kollagenose $\cdot$ Immunsuppression . Interphasendermatitis

\section{More Than a Butterfly Rash-A Guide to the Diversity of Cutaneous Lupus Erythematosus. Pathogenesis, Clinical Picture, Treatment}

\section{Abstract}

The plethora of manifestations of cutaneous lupus erythematosus (CLE) can occur with or without concomitant systemic lupus erythematosus (SLE) for which regular screening is mandatory. Female sex, genetic predisposition, sun exposure, smoking and some medications serve as risk factors for LE. The most important CLE forms are the acute-cutaneous LE (e.g., butterfly rash, generalized macular-papular rash, enoral), the subacute-cutaneous LE (e.g., annular) and the chronic-cutaneous LE (e.g., scaring discoid lesions, panniculitis, chilblain-LE). Diagnosis is primarily based upon the clinical picture and its histopathology. Results of autoimmune serology and direct immunofluorescence can be helpful. For mild forms of CLE local therapy is often sufficient. If not, hydroxychloroquine is the first choice of systemic therapy in addition to transient systemic steroids. For recalcitrant cases additional therapy with methotrexate, retinoids, dapsone, mycophenolate mofetil, azathioprine, thalidomide, belimumab and rituximab may be used. All CLE therapies are off-label. An update of conventional vaccinations is advisable, if possible, to be performed before starting immunosuppressive therapies. To objectively verify the therapeutic response, the periodic scoring using the RCLASI (Revised CLE Disease Area and Severity Index) is recommended. Preventive sun protection (cream, clothing, travel destinations) is of pivotal importance, since intense UV-exposure can provoke disease flares. $L E$ is no contraindication against a pregnancy. However, pregnancy should not start during a disease flare since it increases the risk for adverse outcomes for the fetus and mother. Steroids, hydroxychloroquine, dapsone and azathioprine serve as suitable therapies during pregnancy.

Keywords

Systemic lupus erythematosus · Autoimmunity - Connective tissue disease . Immunosuppression · Inteface dermatitis perkeratose $\mathrm{zu}$ dicken Läsionen mit anhaftender Schuppung kommt.

Andere Erscheinungsformen des CCLE an der Haut sind z.B. die $\mathrm{Lu}$ pus-Pannikulitis (•Abb.6), der „mucosal $C C L E^{“}$ oder der an Frostbeulen erinnernde und akral (Finger, Zehen, Nase, Ohren) auftretende Chilblain-Lupus (- Abb. 7).

Der CCLE hat eine geringe Wahrscheinlichkeit (ca. $5 \%$ ), im weiteren Verlauf einen SLE zu entwickeln. Der SCLE ist überdurchschnittlich lichtempfindlich und ebenfalls durch schuppende, erythematöse, jedoch meist anulär angeordnete Plaques gekennzeichnet (anulärer SCLE; - Abb. 8). Eine weitere Spielart des SCLE ist der psoriasiforme SCLE.

Die Herde des SCLE entwickeln sich rascher als beim CCLE, gelegentlich so rasch, dass die Schuppung als Zeichen der Chronizität fehlt. SCLE-Herde treten ebenfalls bevorzugt in den UV-exponierten Arealen auf. Auch die Histologie entspricht im Wesentlichen abgesehen von der Vernarbung der des CCLE.
Der SCLE ist häufiger (ca. 25\%) mit einem SLE assoziiert als der CCLE. Eine deutliche Assoziation besteht auch mit Ro/SSA bzw. La/SSB-Autoantikörpern.

Die Erscheinungsformen des akut kutanen LE (ACLE) umfassen u. a. das weithin bekannte Schmetterlingserythem, welches lange Zeit unkritisch als Synonym für „Hautlupus“ und „SLE“ gesehen wurde. Wie wir mittlerweile wissen, gibt es viele unterschiedliche Arten des kutanen LE. Und: Auch ein Schmetter- 

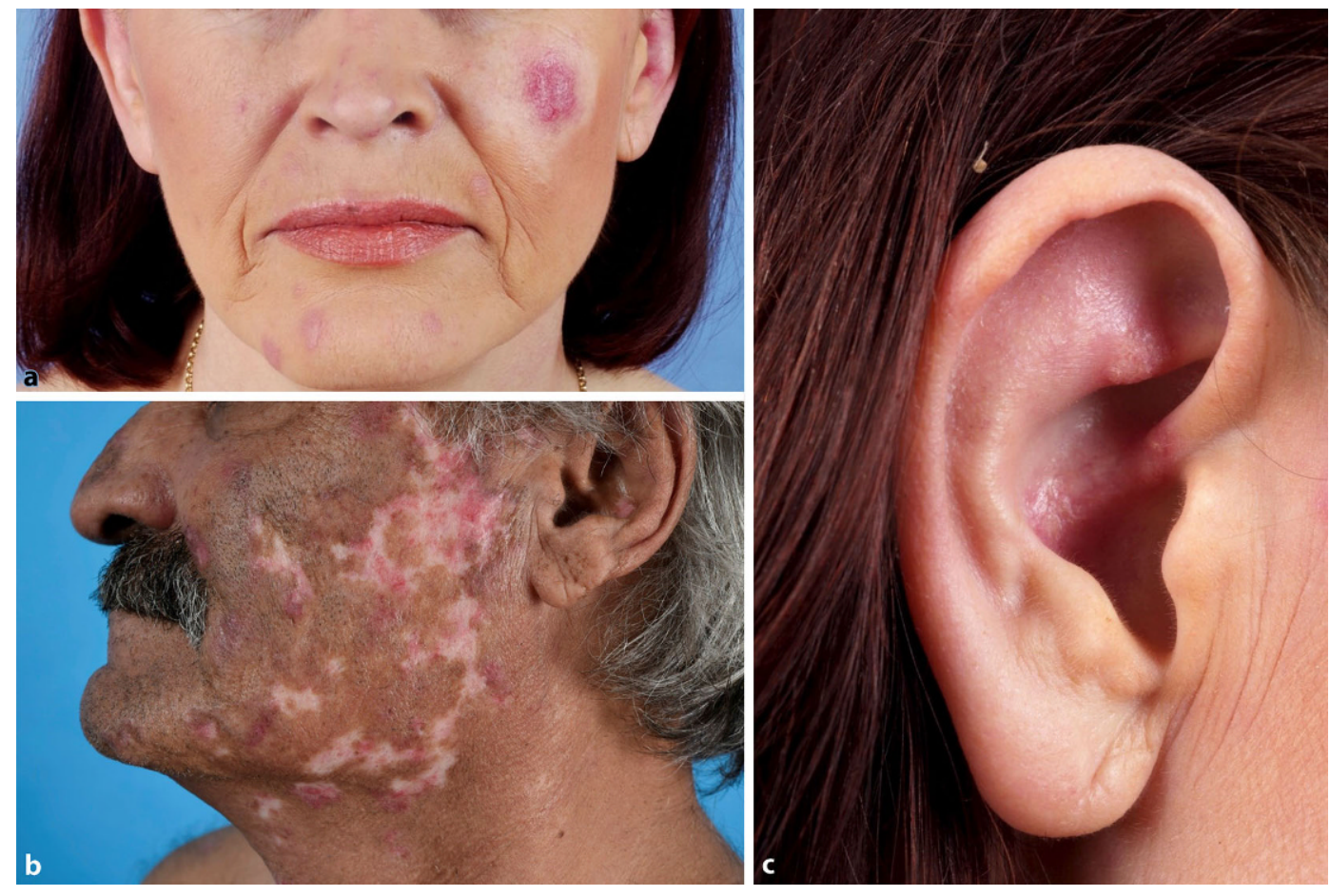

Abb. $2<$ a Limitierter chronisch-diskoider Lupus erythematodes (CDLE). b Limitierter CDLE. c Limitierter CDLE

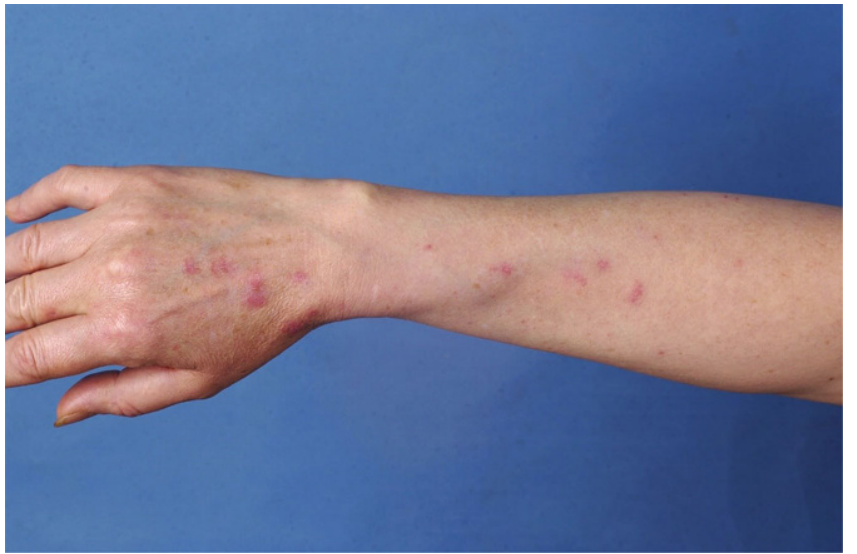

Abb. $3<$ Disseminierter chronischdiskoider Lupus erythematodes

lingserythem kann solitär, ohne SLE, auftreten.

Beim Schmetterlingserythem (auch „butterfly rash“ genannt; • Abb. 9a) handelt es sich um eine binnen weniger Wochen auftretende zentrofaziale Rötung im Bereich der Malarregion, welche auf die Nase übergreift und im Gegensatz zur Dermatomyositis die Augenlider in der Regel ausspart. Ödem und Schuppung können vorkommen.

Zum ACLE werden auch das generalisiert makulopapulöse Exanthem (- Abb. 9b), die charakteristischen entzündlichen Plaques der Fingerstreckseiten (wobei typischerweise die Haut über den Gelenken ausgespart sind, auch dies im Gegensatz zur Dermatomyositis, wo diese typischerweise betroffen sind), die meist palmoplantar auftretende bullöse Form des ACLE, der an eine toxischepidermale Nekrolyse erinnernde „TENlike lupus“(• Abb.9c) sowie der „mucosal $A C L E^{“}$ (• Abb.9d) gezählt. Die Formen des ACLE heilen in der Regel narbenlos ab. Der ACLE ist häufig Ausdruck eines SLE (ca. 75\%).

Eine Sonderform des CLE ist das sog. Rowell-Syndrom, bei dem sich $\mathrm{zu}$ einem ACLE oder CCLE anulär-multiformeartige Läsionen gesellen, die sehr an ein Erythema exsudativum multiforme erinnern.
Der intermittierend kutane Lupus erythematodes (ICLE; auch Lupus tumidus genannt) nimmt insofern eine Sonderstellung ein, als seine Klinik auch bei längerem Bestehen keine epidermale Komponente zeigt (entzündliches Infiltrat liegt tiefer), ganz besonders lichtempfindlich ist und ausgenommen selten in einen SLE übergeht. Die Klassifikation des ICLE ist seit Jahren Anlass zur Diskussion in Fachkreisen, die ihn entweder zum CCLE zuordnen [29] oder als eigene Kategorie führen [30].

$\mathrm{Da}$ Autoantikörper plazentagängig sind, können Neugeborene von Müttern, die an einem LE leiden, selbst daran erkrankt sein (neonataler LE; s. unten).

Unter dem Begriff „drug-induced lupus" (DIL; - Abb. 10; [22]) werden sowohl der medikamentös getriggerte CLE als auch das das "lupus-like syndrome“ (vorübergehendes SLE-ähnliches Bild, das meist nach Absetzen des kausalen Medikaments von alleine in Remission geht und selten Therapie benötigt) bzw. auch das Auftreten eines manifesten SLE (durch Demaskieren eine latenten SLE oder Hervorrufen eines Schubes eines bereits bekannten SLE) subsummiert. Das „lupus-like syndrome“ ist im Regelfall milder als ein idiopathischer SLE. 


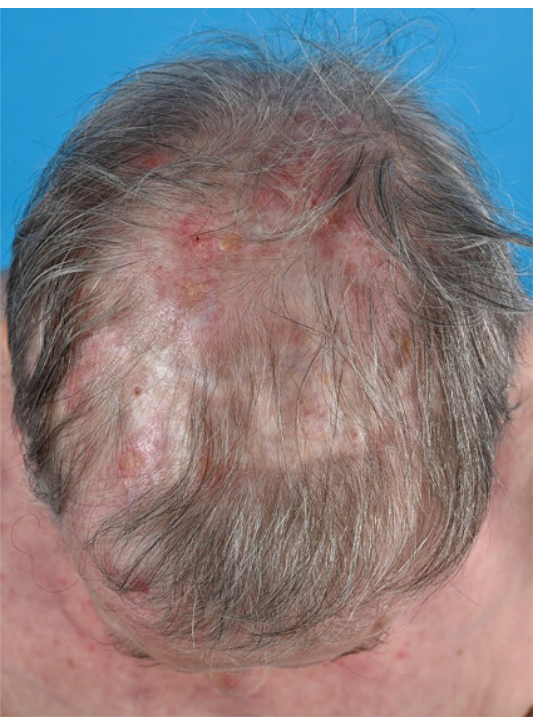

Abb. $4 \Delta$ Chronisch-diskoider Lupus erythematodes, vernarbende Alopezie

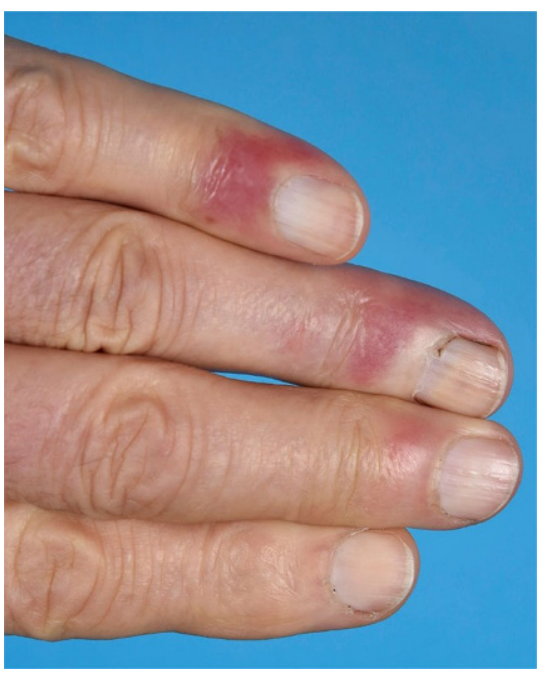

Abb. $7 \Delta$ Chilblain-Lupus

$\mathrm{Zu}$ den unspezifischen kutanen Manifestationen, die im Rahmen eines LE auftreten können, zählt das Raynaud-Syndrom (• Abb. 11): Dieses ist definiert als spontan oder durch Triggerfaktoren wie Kälte oder Stress induzierte Spasmen der Fingerarterien mit triphasischer Hautreaktion: zuerst weiß (Anämie), dann blau (Hypoxie), dann rot (reaktive Hyperämie). Ebenso gehören in diese Kategorie die Alopecia areata, das diffuse Effluvium (• Abb. 12), die Livedo racemosa (• Abb. 13, durch Hypoxie ausgelöste rundlich livide Veränderungen, deren Kreise im Gegensatz zur physiologischen Livedo reticularis nicht geschlossen sind), Veränderungen der Nagelfalz-

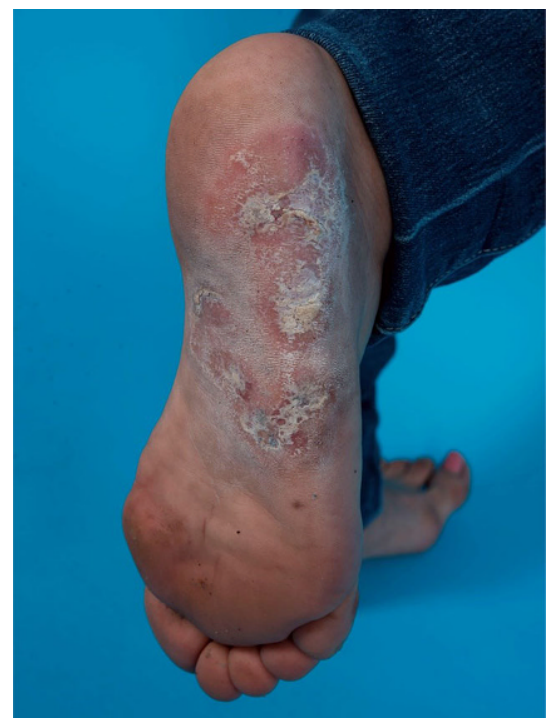

Abb. 5 A Hypertropher chronisch-diskoider Lupus erythematodes

gefäße (Kapillarmikroskopie) sowie eine leukozytoklastische Vaskulitis.

Die Organmanifestationen eines SLE sind mannigfaltig. Sie umfassen Nephritis, Arthritis, Pneumonitis, Myokarditis, Hepatitis und vieles mehr.

Overlaps und assoziierte Syndrome: Jede Kollagenose kann grundsätzlich mit anderen Kollagenosen klinisch und immunserologisch überlappen, insbesondere dem SLE. Dies bedeutet für die klinische Routine, dass die Anamnese und der klinische Blick auch Zeichen der Dermatomyositis, der systemischen Sklerose, des Sjögren-Syndroms und der rheumatoiden Arthritis erkennen sollten, um hier rechtzeitig eine weitergehende Abklärung und ggf. eine entsprechende Therapie einleiten zu können. Im weitesten Sinne kann man hier auch das AntiphospholipidAntikörper-Syndrom (APLAS) nennen. Hier kann es durch AntiphospholipidAutoantikörper (Cardiolipin, Beta-2Glykoprotein I, Lupushemmstoff) neben Schwangerschaftskomplikationen (s. unten) zu einer Embolie im venösen oder arteriellen Gefäßbett kommen (-Abb. 14).

\section{Diagnose}

Die Diagnose eines CLE erfolgt anhand des geschilderten klinischen Bildes und der Ergebnisse von Hautbiopsien (His-
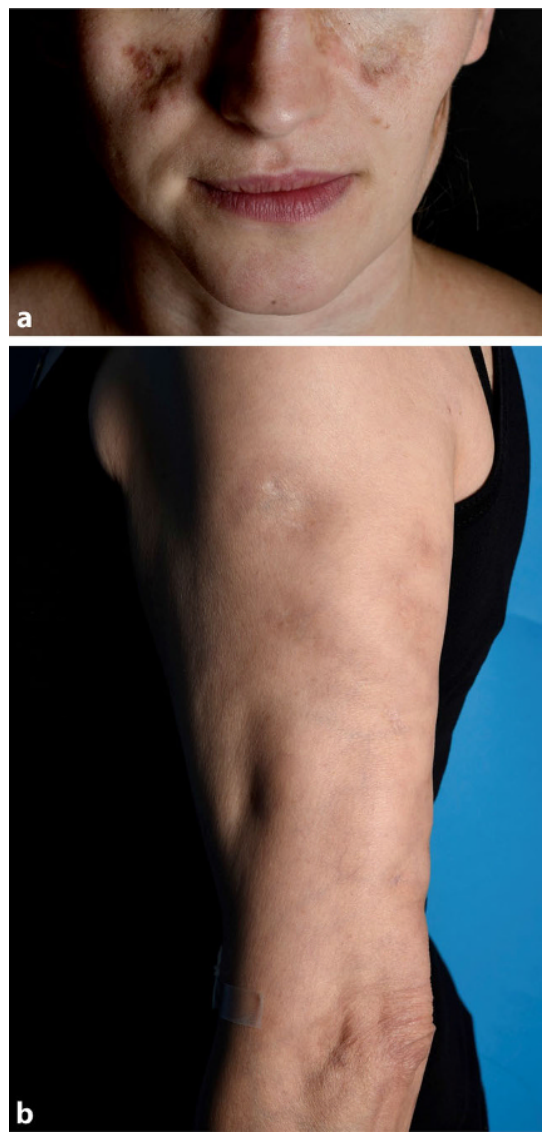

Abb. 6 \ a LE-Pannikulitis. b LE-Pannikulitis

tologie bzw. direkte Immunfluoreszenz, DIF). Die Histologie der Formen des CLE ist entsprechend der heterogenen Klinik unterschiedlich; dazu kommt, dass die histologischen Befunde von der Dauer der Läsion abhängig und dadurch variabel sind. Ein typischer CCLE ist gekennzeichnet durch eine Differenzierungsstörung, ein „follicular plugging“, eine Verdickung der Balsamembranzone sowie ein perivaskuläres und periadnexielles Rundzellinfiltrat. Das typische Bild des SCLE besteht aus Interphasendermatitis, Aufsplitterung der Basalmembranzone und einem perivaskulären Rundzellinfiltrat, wohingegen der ACLE ein perivaskuläres Rundzellinfiltrat mit meist noch geringer Interphasendermatitis zeigt. Die LE-Pannikulitis ist primär lobulär und zeigt ein lymphozytäres Infiltrat, das zur Nekrose der Adipozyten führen kann, was sich klinisch in Eindellungen der Hautkontur ausdrückt. Beim bullösen ACLE besteht das entzündliche Infiltrat vor allem aus neutrophilen Granulo- 


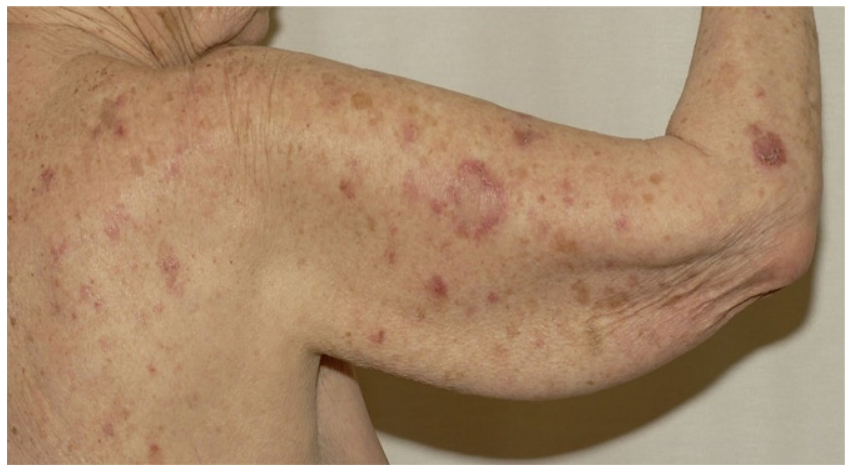

Abb. $8<$ Anulärer subakut-cutaner Lupus erythematodes
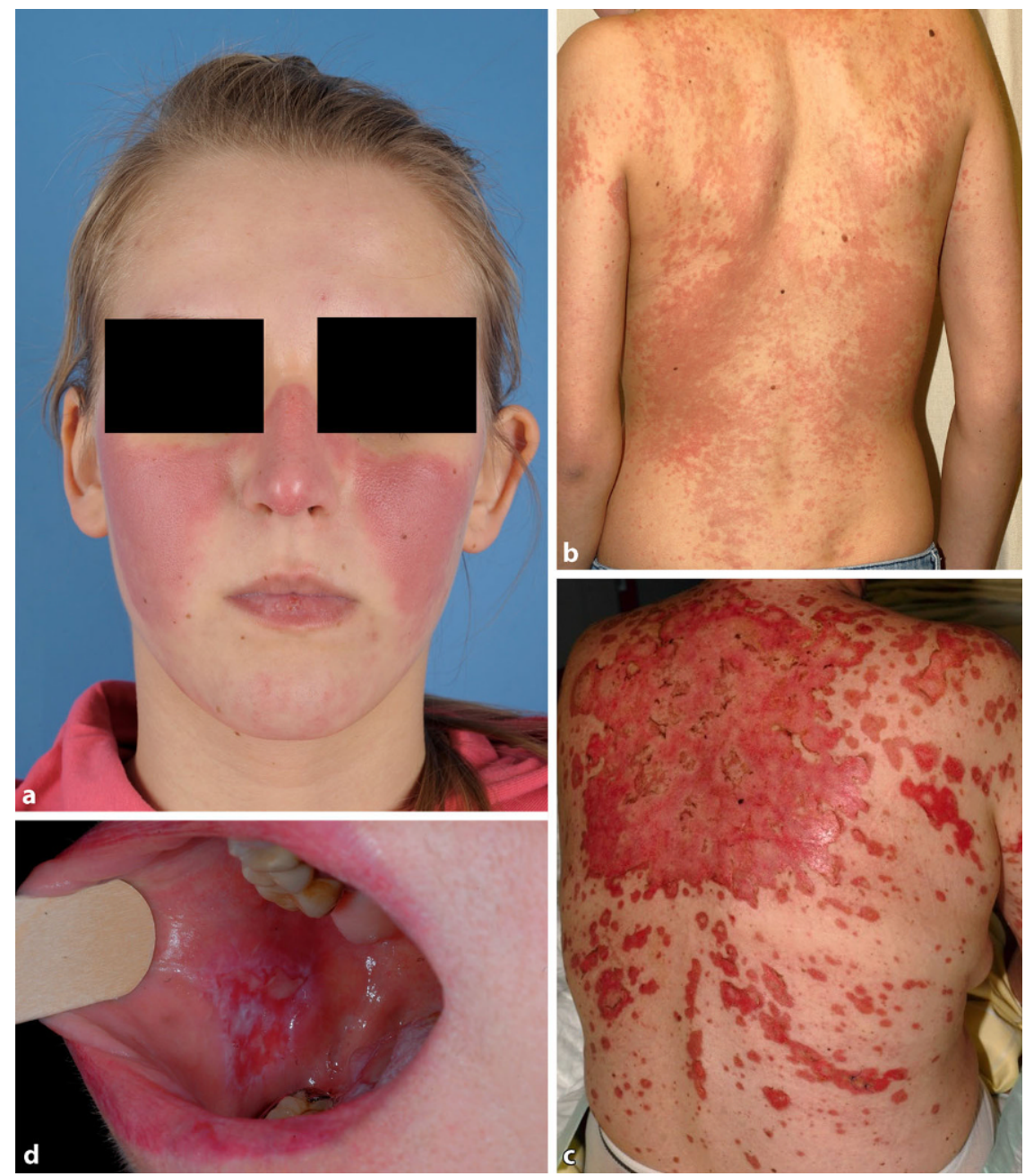

Abb. $9 \Delta$ a Akut-cutaner Lupus erythematodes (ACLE), Schmetterlingserythem. b ACLE, makulopapulöses Exanthem. CACLE, toxic epidermal necrolysis-like. d ACLE, enoral

zyten, der Spalt ist subepidermal, und häufig lassen sich Autoantikörper gegen Kollagen Typ VII nachweisen.

In der DIF, welche zur Vermeidung von falsch-positiven Befunden aus nichtlichtexponierter Haut entnommen werden sollte, zeigen sich granuläre Ablage- rungen von vor allem IgG (aber auch IgM und C3) entlang der Basalmembranzone.

Die Diagnose des SLE erfolgte bis vor Kurzem hauptsächlich über die aus 1997 stammenden ACR-Kriterien des American College of Rheumatology [31]. Im Jahr 2019 wurden überarbeitete gemeinsame Kriterien des ACR und der europä-

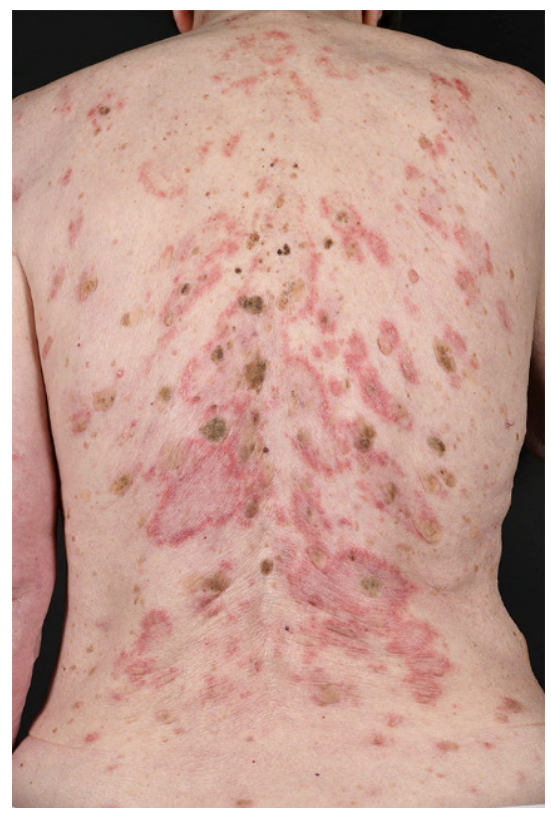

Abb. $10 \triangle$ "Drug-induced lupus" (DIL), Auslöser: Terbinafin

ischen Fachgesellschaft (EULAR) publiziert, die sich möglicherweise durchsetzen werden [32]. Auch diese sind wie die ACR-Kriterien eine Kombination aus klinischen und laborchemischen Kriterien, in denen die dermatologischen Manifestationen jedoch präziser (Alopezie, orale Ulzera, ACLE, SCLE, CDLE) abgebildet sind als bei den ACR-Kriterien.

Charakteristisch für den DIL sind $\mathrm{Au}-$ toantikörper gegen Histone. Im Labor zeigt der DIL im Vergleich zum SLE seltener Zytopenien oder Erniedrigungen von Komplementfaktoren.

Screening und Scoring. Auch Patienten mit zunächst rein kutanem LE sollten regelmäßig auf das Vorliegen eines sich entwickelnden SLE untersucht werden, da dies mit oft nur geringen Symptomen wie z.B. chronischer Erschöpfung klinisch auffallen kann; dies trifft besonders auf die Nephritis zu, die im Gegensatz zu anderen Organbeteiligungen (z.B. Pleuritis) nahezu symptomlos ist. $\mathrm{Zu}$ solch einem Screening zählen neben einer gründlichen Anamnese Blutbefunde (BB, Diff-BB, Chemie, Gerinnung, ANA, AAK gegen ENA und dsDNA, Antiphospholipid AK, Lupushemmstoff) und Harnbefunde (Mikroalbumin-Kreatinin-Ratio, 


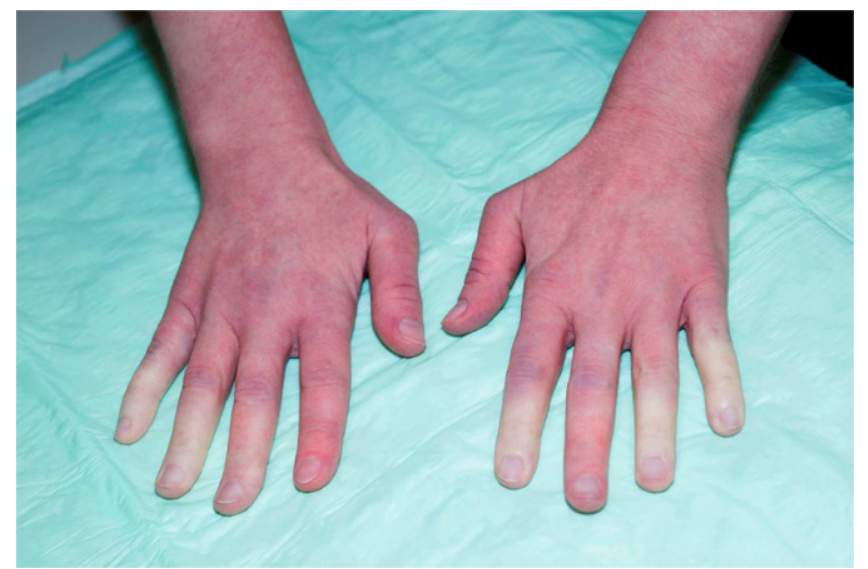

Abb. $11 \triangleleft$ Raynaud-Syndrom

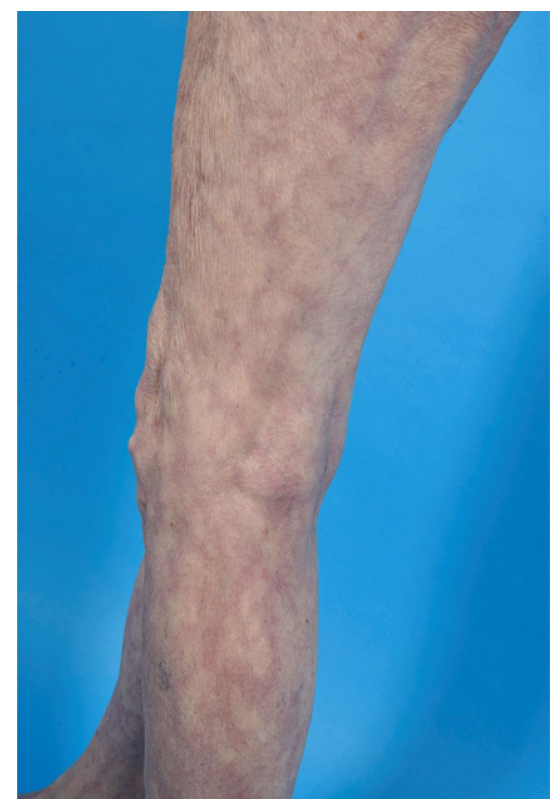

Abb. $13 \Delta$ Livedo racemosa

Protein-Kreatinin-Ratio, Harnsediment auf dysmorphe Erythrozyten).

Zur klinischen Quantifizierung der Ausdehnung eines CLE eignet sich der RCLASI (Revised Cutaneous Lupus Erythematosus Disease Area and Severity Index; [33]). Dieser beschreibt in allen anatomischen Regionen (inkl. Schleimhäute und Capillitium) das Ausmaß der aktiven Hautveränderungen (Erythem, Schuppung) sowie des bereits irreversibel eingetretenen Schadens (Vernarbung, Dyspigmentierung); man erhält am Ende zwei Ziffern, welche „activity“ und „damage“ benennen und zusammen den „total score" ergeben. Ziel einer Therapie ist also, die Ziffern der Aktivität auf null zu drücken. Neben dem RCLASI empfiehlt es sich, eine genaue Fotodokumentation von Veränderungen durchzuführen, um im Verlauf Detailvergleiche durchführen zu können.

\section{Prävention und Therapie}

Konsequentester Sonnenschutz stellt die zentrale Säule der Prävention dar. Die Information über den Zusammenhang zwischen UV-Exposition und Risiko eines LE-Schubs muss fixer Bestandteil der Patienteninformation sein. Präventionsmaßnahmen (Sunblocker, UVSchutzkleidung, Wahl der Reiseziele) sollten regelmäßig besprochen werden.

Die Therapie des isoliert kutanen LE bzw. eines SLE, dessen einzig betroffenes Organ die Haut ist (serokutaner SLE), wird meist von Dermatologen durchgeführt. In Bezug auf die Therapie des CLE sei auch auf die gültige S2k-Leitlinie verwiesen [34].

Bei geringer Ausprägung des CLE ist eine Lokaltherapie (lokale Steroide [33] und Calcineurininhibitoren [35-37]) ausreichend; bei länger andauernder Lokaltherapie sollte Calcineurininhibitoren aufgrund des fehlenden atrophogenen Potenzials gegenüber Lokalsteroiden der Vorzug gegeben werden.

Neben einem systemischen Kortisonstoß (0,5-1,0 mg Prednisolon-Äquivalent/kg KG) als Akuttherapie ist die etablierteste steroidsparende Systemtherapie jene mit dem Antimalariamittel Hydroxychloroquin [38] in einer Dosierung von bis $\mathrm{zu} 6,5 \mathrm{mg} / \mathrm{kgKG}$ pro Tag (Tabletten zu je $200 \mathrm{mg}$ ), welche in der aktuellen S2k-Leitlinie auch als einzige steroidsparende First-line-Therapie geführt wird. Die wichtigsten Nebenwirkungen

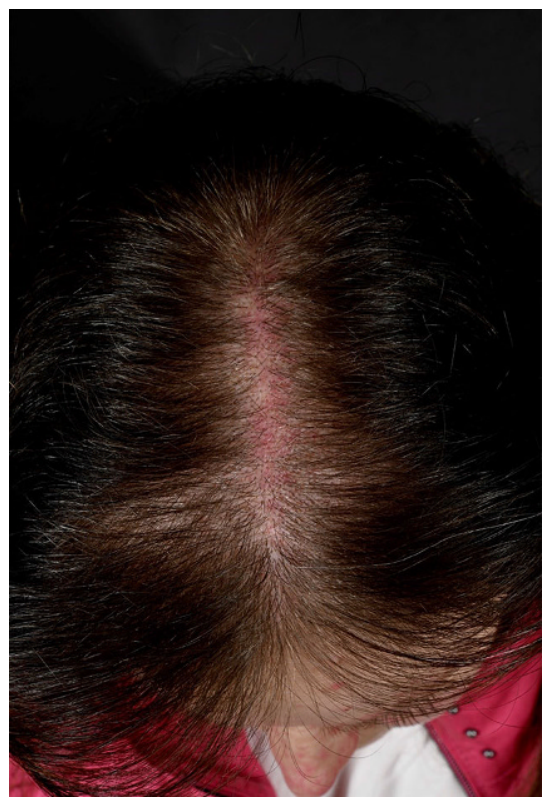

Abb. $12 \Delta$ Diffuses Effluvium bei Schub eines akut-cutanen Lupus erythematodes

sind Ablagerungen in der Netzhaut sowie im Myokard, EKG-Veränderungen, Kopfschmerz, abdominelle Beschwerden (Inappetenz, Übelkeit, Diarrhoe) und Stimmungsschwankungen. Als seltene Nebenwirkung ist eine Typ- IVAllergie beschrieben [39]. Regelmäßige ophthalmologische Kontrollen sind deshalb so wichtig, da Ablagerungen möglichst frühzeitig erkannt werden sollten, noch ehe subjektiv wahrnehmbare Sehstörungen auftreten. Dies ist umso wichtiger, als man trotz sofortiger Therapieunterbrechung auf die lange Halbwertszeit von Hydroxychloroquin von etwa 2 Monaten Rücksicht nehmen muss.

Rauchen sollte unbedingt beendet werden, da es nicht nur (s. oben) ein Risikofaktor für die Entstehung eines CLE ist, sondern zudem auch die therapeutische Wirksamkeit von Hydroxychloroquin schwächt [40].

Falls Hydroxychloroquin nach einigen Monaten zu keinem ausreichenden Erfolg führt, ist das nächste Immunsuppressivum der Wahl Methotrexat (Second-line-Therapie; [41]). Ist auch mit Methotrexat keine Remission zu erzielen oder besteht eine Kontraindikation, kommen die beiden anderen Second-line-Optionen Retinoide ([42]; insbesondere beim hypertrophen CDLE) 


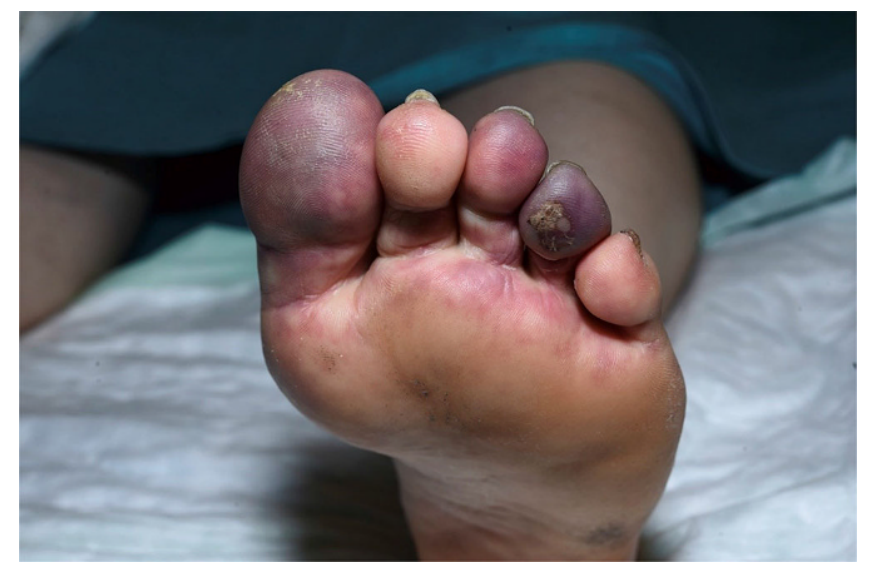

Abb. $14<$ Antiphospholipid-Antikörper-Syndrom

und Dapson ([43]; insbesondere beim bullösen ACLE) zum Einsatz.

Weitere Therapeutika (alle Third-lineTherapien) stellen Mycophenolat-Mofetil [44], Thalidomid (strengste duale Kontrazeption; [45]) sowie Azathioprin (während Schwangerschaft einsetzbar; [46]) dar.

Das einzige derzeit für den SLE zugelassene Biologikum, Belimumab (Hemmer des B-lymphozyte stimulators $[\mathrm{BlyS}]=\mathrm{B}$-Cell Activating Factor [BAFF]), hat in ersten Beobachtungen eine gute Wirkung auf kutane LE-Formen gezeigt [47]. In Einzelfällen können Immunglobuline (i.v. oder s.c.) oder Rituximab eine sinnvolle Option darstellen, insbesondere dann, wenn ein SLE im Hintergrund steht [48].

Die o.g. Reihung (First-/Second-/ Third-line) ist als Empfehlung zu verstehen für den Fall, dass die Haut die einzige Endorganaktivität darstellt. Im klinischen Alltag kommt es regelmäßig vor, dass neue Aspekte, die zusätzlich zum CLE auftreten, eine andere Therapiegewichtung erfordern (z. B. MMF bei Nierenbeteiligung oder Lungenbeteiligung, Azathioprin bei Schwangerschaft, Dapson bei Neutrophilen-getragener Vaskulitis usw.).

Falls es die Akuität der Erkrankung zulässt, sollte noch vor Beginn einer Immunsuppression eine Aktualisierung der Impfungen durchgeführt werden, spätestens parallel zur begonnen Therapie, um das erhöhte Infektionsrisiko zu minimieren.

Aufgrund des Sonnenschutzverhaltens der Patienten sollte regelmäßig eine Kontrolle des Vitamin-D-Spiegels er- folgen und entsprechend suffizient und nachkontrolliert ein Mangel ausgeglichen werden.

Beim medikamentös induzierten Lupus erythematodes ist die wichtigste Maßnahme die Identifikation des kausalen Agens und dessen Absetzen. Sollte dies nicht ausreichen, ist therapeutisch gemäß dem idiopathischen LE vorzugehen.

Medicolegaler Aspekt. Wichtig ist $\mathrm{zu}$ betonen, dass keine der genannten Systemtherapien für die Therapie des CLE zugelassen ist und man sich als Dermatologe hier im off-label bewegt, weshalb eine besonders gründliche, auch schriftliche, Aufklärung erfolgen sollte, wofür substanzspezifische Aufklärungsreverse existieren.

\section{Lupus erythematodes und Schwangerschaft}

Ein CLE, aber auch ein SLE, stellt keine grundsätzliche Kontraindikation gegen eine Schwangerschaft dar, jedoch sollte diese mit einer auf Risikoschwangerschaften spezialisierten Ambulanz geplant und von dieser begleitet werden. Therapeutisch stehen während der Schwangerschaft Steroide, Hydroxychloroquin, Azathioprin, Immunglobuline (i.v. oder s.c.) und Dapson zur Verfügung. Eine Nutzen-Risiko-Abwägung muss erfolgen.

Keinesfalls sollte eine Schwangerschaft während eines Krankheitsschubs stattfinden, da dies die Komplikationsrate für Mutter und Kind erhöht. Kommt es zu einer Schwangerschaft während eines
LE-Schubs, sollte unverzüglich die $\mathrm{Zu}$ weisung an ein spezialisiertes Zentrum erfolgen.

Exemplarisch sei auf die folgenden beiden Problemkonstellationen hingewiesen: Das Antigen zu mütterlichen Ro/SSA- bzw. La/SSB-Autoantikörpern ist noch auf dem fetalen Reizleistungssystem exprimiert und führt bei ca. $2 \%$ der Feten/Neugeborenen zum „cardiac neonatal lupus erythematosus" (CNLE). Das klinische Spektrum reicht von Herzrhythmusstörungen bis hin zum Tod (ca. $20 \%$ der C-NLE-Fälle). Etwa $70 \%$ der C-NLE-Fälle benötigen einen Schrittmacher [49-51]. ScreeningMethode der Wahl ist das fetale EKG (erweiterte Methode des Tocogramms). Die therapeutischen Optionen reichen von plazentagängigen Steroiden bis hin zur Apherese [52]. Ro/SSA- bzw. La/SSBpositive Schwangere sollten auch ohne Klinik präventiv Hydroxychloroquin erhalten [53].

Ein APLAS kann Aborte, Frühgeburten und (Prä-)Eklampsie auslösen. Mütter mit positiven Antiphospholipid-Antikörpern sollten auch ohne stattgehabtes klinisches Ereignis präventiv $100 \mathrm{mg}$ Acetylsalicylsäure (ASS) erhalten [53].

\section{Conclusio}

Klinik und Histopathologie des kutanen Lupus erythematodes sind vielfältig, was im klinischen Alltag eine Herausforderung darstellen kann. Dies gilt auch für das therapeutische Armamentarium, das breit ist und an die individuelle Konstellation des Patienten angepasst werden muss. Ein Screening (Blut- und Harnbefunde) auf einen parallel auftretenden systemischen Lupus erythematodes ebenso wie auf Overlap-Syndrome sollte regelmäßig und standardisiert erfolgen. Ein Lupus erythematodes stellt keine Kontraindikation für eine Schwangerschaft dar, zumal ausreichend sichere und effektive Therapien existieren. 


\section{Korrespondenzadresse}

\section{Dr. P. M. Heil}

Kollagenosen-Ambulanz, Universitätsklinik für Dermatologie, Medizinische Universität Wien Währinger Gürtel 18-20, 1090 Wien, Österreich peter.heil@meduniwien.ac.at

Funding. Open access funding provided by Medical University of Vienna.

\section{Einhaltung ethischer Richtlinien}

Interessenkonflikt. P. M. Heil gibt an, dass kein Interessenkonflikt besteht.

Für diesen Beitrag wurden von den Autoren keine Studien an Menschen oder Tieren durchgeführt. Für die aufgeführten Studien gelten die jeweils dort angegebenen ethischen Richtlinien.

Open Access. Dieser Artikel wird unter der Creative Commons Namensnennung 4.0 International Lizenz veröffentlicht, welche die Nutzung, Vervielfältigung, Bearbeitung, Verbreitung und Wiedergabe in jeglichem Medium und Format erlaubt, sofern Sie den/die ursprünglichen Autor(en) und die Quelle ordnungsgemäß nennen, einen Link zur Creative Commons Lizenz beifügen und angeben, ob Änderungen vorgenommen wurden.

Die in diesem Artikel enthaltenen Bilder und sonstiges Drittmaterial unterliegen ebenfalls der genannten Creative Commons Lizenz, sofern sich aus der Abbildungslegende nichts anderes ergibt. Sofern das betreffende Material nicht unter der genannten Creative Commons Lizenz steht und die betreffende Handlung nicht nach gesetzlichen Vorschriften erlaubt ist, ist für die oben aufgeführten Weiterverwendungen des $\mathrm{Ma}$ terials die Einwilligung des jeweiligen Rechteinhabers einzuholen.

Weitere Details zur Lizenz entnehmen Sie bitte de Lizenzinformation auf http://creativecommons.org/ licenses/by/4.0/deed.de.

\section{Literatur}

1. Durosaro 0 et al (2009) Incidence of cutaneous lupus erythematosus, 1965-2005: a populationbased study. Arch Dermatol 145:249-253

2. Jarukitsopa $S$ et al (2015) Epidemiology of systemic lupus erythematosus and cutaneous lupus erythematosus in a predominantly white population in the United States. Arthritis Care Res 67:817-828

3. Wenzel J et al (2005) Scarring skin lesions of discoid lupus erythematosus are characterized by high numbers of skin-homing cytotoxic lymphocytes associated with strong expression of the type I interferon-induced protein $\mathrm{MxA}$. Br J Dermato 153:1011-1015

4. Grassi M, Capello F, Bertolino L, Seia Z, Pippione M (2009) Identification of granzyme B-expressing CD-8-positive T cells in lymphocytic inflammatory infiltrate in cutaneous lupus erythematosus and in dermatomyositis. Clin Exp Dermatol 34:910-914
5. ReichA,MeurerM, Viehweg A,MullerDJ (2009) Narrow-band UVB-induced externalization of selected nuclear antigens in keratinocytes: implications for lupus erythematosus pathogenesis. Photochem Photobiol 85:1-7

6. Scholtissek B et al (2017) Immunostimulatory endogenous nucleic acids drive the lesional inflammation in cutaneous lupus rythematosus. JInvest Dermatol 137:1484-1492

7. Sarkar MK et al (2018) Photosensitivity and type I IFN responses in cutaneous lupus are driven by epidermalderived interferon kappa. Ann Rheum Dis 77:1653-1664

8. Zhang Y-P, Wu J, Han Y-F, Shi Z-R, Wang L (2017) Pathogenesis of cutaneous lupus erythema associated with and without systemic lupus erythema. Autoimmun Rev 16:735-742

9. Zahn S et al (2011) Evidence for a pathophysiological role of keratinocyte-derived type III interferon (IFN $\lambda$ ) in cutaneous lupus erythematosus. J Invest Dermatol 131:133-140

10. Wenzel J et al (2005) Enhanced type I interferon signaling promotes Th1-biased inflammation in cutaneous lupus erythematosus. J Pathol 205:435-442

11. Lauffer $F$ et al (2018) Type I immune response induces keratinocyte necroptosis and is associated with interface dermatitis. J Invest Dermatol 38:1785-1794

12. Farkas L et al (2001) Plasmacytoid dendritic cells (natural interferon-alpha/beta-producing cells) accumulate in cutaneous lupus erythematosus lesions. Am J Pathol 159:237-243

13. Safi R et al (2019) Investigating the presence of neutrophil extracellular traps in cutaneous lesions of different subtypes of lupus erythematosus. Exp Dermatol 28:1348-1352

14. Whitacre CC (2001) Sex differences in autoimmune disease. Nat Immunol 2:777-780

15. Durosaro $O$ et al (2009) Incidence of cutaneous lupus erythematosus, 1965-2005: a populationbased study. Arch Dermatol 145:249-253

16. Khan D, Ansar Ahmed S (2016) The immune system is a natural target for estrogen action: opposing effects of estrogen in two prototypical autoimmune diseases. Front Immunol 6:635

17. Hersh AO, Arkin LM, Prahalad S (2016) Immunogenetics of cutaneous lupus erythematosus. Curr Opin Pediatr 28:470-475

18. Peschke K et al (2014) Deregulated type I IFN response in TREX1-associated familial chilblain lupus. J Invest Dermatol 134:1456-1459

19. Ravenscroft JC et al (2011) Autosomal dominan inheritance of a heterozygous mutation in SAMHD1 causing familial chilblain lupus. Am J Med Genet A 155A:235-237

20. König N et al (2017) Familial chilblain lupus due to a gain-of-function mutation in STING. Ann Rheum Dis 76:468-472

21. Kuhn A et al (2014) Influence of smoking on disease severity and antimalarial therapy in cutaneous lupus erythematosus: analysis of 1002 patients from the EUSCLE database. $\mathrm{Br} J$ Dermatol 171:571-579

22. Vaglio A et al (2018) Drug-induced lupus: traditional and new concepts. Autoimmun Rev 17:912-918

23. Shovman $O$ et al (2018) Diverse patterns of antiTNF- $a$-induced lupus: case series and review of the literature. Clin Rheumatol 37:563-568

24. Levine $D$ et al (2010) Cutaneous lupus erythematosus and anti-TNF-a therapy: a case report with review of the literature. J Drugs Dermato 9:1283-1287
25. Quaglia Met al (2021) Viral infections and systemic lupus erythematosus: new players in an old story. Viruses 13:277

26. Battaglia M, Garrett-Sinha LA (2020) Bacterial infections in lupus: roles in promoting immune activation and in pathogenesis of the disease. J Transl Autoimmun 4:100078

27. Fernandez-Ruiz R et al (2020) COVID-19 in patients with systemic lupus erythematosus: lessons learned from the inflammatory disease. Transl Res. https://doi.org/10.1016/j.trsl.2020.12.007

28. Cornelius R, Pawlak CR et al (2003) Flares in patients with systemic lupus erythematosus are associated with daily psychological stress. Psychother Psychosom 72:159-165

29. Sontheimer RD (1997) The lexicon of cutaneous lupus erythematosus-A review and personal perspective on the nomenclature and classification of the cutaneous manifestations of lupus erythematosus. Lupus 6:84-95

30. Kuhn A, Landmann A (2014) The classification and diagnosis of cutaneous lupus erythematosus. Jutoimmun 48-49:14-19

31. Hochberg MC (1997) Updating the American College of Rheumatology revised criteria for the classification of systemic lupus erythematosus. Arthritis Rheum 40:1725

32. Aringer $M$ et al (2019) 2019 European League Against Rheumatism/American College of Rheumatology classification criteria for systemic lupus erythematosus. Arthritis Rheumato 71:1400-1412

33. Kuhn A et al (2010) Revised Cutaneous Lupus Erythematosus Disease Area and Severity Index (RCLASI): a modified outcome instrument for cutaneous lupus erythematosus. $\mathrm{Br} J$ Dermatol 163:83-92

34. AWMF (2020) S2k-Leitlinie "Diagnostik und Therapie des kutanen Lupus erythematodes" (AWMF-Registernummer 013-060)

35. Roenigk HH et al (1980) Discoid lupus erythematosus: diagnostic features and evaluation of topical corticosteroid therapy. Cutis 25:281-285

36. Tzung TY et al (2007) Tacrolimus vs. clobetasol propionate in the treatment of facial cutaneous lupus erythematosus: a randomized, doubleblind, bilateral comparison study. Br J Dermatol 156:191-192

37. Tlacuilo-Parra A et al (2005) Pimecrolimus $1 \%$ cream for the treatment of discoid lupus erythematosus. Rheumatology (Oxford) 44:1564-1568

38. Yokogawa $\mathrm{N}$ et al (2017) Effects of Hydroxychloroquine in patients with cutaneous lupus erythematosus: a multicenter, double-blind, randomized, parallel-group trial. Arthritis Rheumatol 69:791-799

39. Borik L et al (2019) Type IV allergy to antimalarials can mimic cutaneous manifestations of lupus erythematosus. J Eur Acad Dermatol Venereol 33:e94-e96

40. Chasset F, Frances C, Barete S, Amoura Z, Arnaud L (2015) Influence of smoking on the efficacy of antimalarials in cutaneous lupus: a meta-analysis of the literature. JAm Acad Dermatol 72:634-639

41. Wenzel J, Brahler S, Bauer R, Bieber T, Tuting $T$ (2005) Efficacy and safety of methotrexate in recalcitrant cutaneous lupus erythematosus: results of a retrospective study in 43 patients. $\mathrm{Br} \mathrm{J}$ Dermatol 153:157-162

42. Ruzicka Tetal (1992) Treatment of cutaneous lupus erythematosus with acitretin and hydroxychloroquine. Br JDermatol 127:513-518

43. Klebes $M$ et al (2016) Dapsone as second-line treatment for cutaneous lupus erythematosus? 
retrospective analysis of 34 patients and a review of the literature. Dermatology 232:91-96

44. Sadlier M et al (2012) Mycophenolate mofetil and hydroxychloroquine: an effective treatment for recalcitrant cutaneous lupus erythematosus. J Am Acad Dermatol 66:160-161

45. ChassetF, Tounsi T, CesbronE, Barbaud A, Frances C, Arnaud L (2018) Efficacy and tolerance profile of thalidomide in cutaneous lupus erythematosus: a systematic review and meta-analysis. J Am Acad Dermatol 78:342-350.e4

46. Callen JP, Spencer LV, Burruss JB, Holtman J (1991) Azathioprine. An effective, corticosteroid-sparing therapy for patients with recalcitrant cutaneous lupus erythematosus or with recalcitrant cutaneous leukocytoclastic vasculitis. Arch Dermatol 127:515-522

47. Salle R et al (2020) Belimumab for refractory manifestations of cutaneous lupus: a multicenter, retrospective observational study of 16 patients. J Am Acad Dermatol 83:1816-1819

48. Quelhas da Costa R et al (2018) Assessment of response to $B$-cell depletion using rituximab in cutaneous lupus erythematosus. JAMA Dermatol 154:1432-1440

49. Brucato A et al (2001) Risk of congenital complete heart block in newborns of mothers with anti-Ro/SSA antibodies detected by counterimmunoelectrophoresis: a prospective study of 100 women. Arthritis Rheum 44:1832-1835

50. Izmirly PM et al (2011) Maternal and fetal factors associated with mortality and morbidity in a multiracial/ethnic registry of anti-SSA/Ro associated cardiacneonatal lupus. Circulation 124:1927-1935

51. Brooks P (2015) Fetal manifestations of maternal anti-Ro and La antibodies-more than complete heart block. Australas J Ultrasound Med 18:124-128

52. MiyakataSetal (2001)Therapeutic plasmapheresis for the prevention of congenital complete heart block associated with anti-SS-A/Ro antibody and anti-SS-B/La antibody. Ryumachi 41:726-735

53. Sammaritano LR et al (2020) 2020 American college of rheumatology guideline for the management of reproductive health in rheumatic and musculoskeletal diseases. Arthritis Care Res (Hoboken) 72:461-488

Hinweis des Verlags. Der Verlag bleibt in Hinblick auf geografische Zuordnungen und Gebietsbezeichnungen in veröffentlichten Karten und Institutsadressen neutral.
Hier steht eine Anzeige. Springer 